\title{
Editorial
}

\section{Extreme Learning Machine on High Dimensional and Large Data Applications}

\author{
Zhiping Lin, ${ }^{1}$ Jiuwen Cao, ${ }^{2}$ Tao Chen, ${ }^{3}$ Yi Jin, ${ }^{4}$ Zhan-Li Sun, ${ }^{5}$ and Amaury Lendasse ${ }^{6,7}$ \\ ${ }^{1}$ School of Electrical and Electronic Engineering, Nanyang Technological University, Singapore 639798 \\ ${ }^{2}$ Key Lab for IOT and Information Fusion Technology of Zhejiang, Hangzhou Dianzi University, Zhejiang 310018, China \\ ${ }^{3}$ Department of Visual Computing, Institute for Infocomm Research, Agency for Science, Technology and Research, \\ 1 Fusionopolis Way, No. 21-01 Connexis (South Tower), Singapore 138632 \\ ${ }^{4}$ School of Computer and Information Technology, Beijing Jiaotong University, No. 3 Shangyuancun, Haidian District, \\ Beijing 100044, China \\ ${ }^{5}$ School of Electrical Engineering and Automation, Anhui University, Hefei 230601, China \\ ${ }^{6}$ Department of Mechanical and Industrial Engineering and The Iowa Informatics Initiative, The University of Iowa, \\ Iowa City, IA 52242-1527, USA \\ ${ }^{7}$ Arcada University of Applied Sciences, 00560 Helsinki, Finland \\ Correspondence should be addressed to Zhiping Lin; ezplin@ntu.edu.sg
}

Received 29 March 2015; Accepted 29 March 2015

Copyright (c) 2015 Zhiping Lin et al. This is an open access article distributed under the Creative Commons Attribution License, which permits unrestricted use, distribution, and reproduction in any medium, provided the original work is properly cited.

Extreme learning machine (ELM) has been developed for single hidden layer feedforward neural networks (SLFNs) training in the past decade. ELM theory claims that the hidden node parameters of SLFNs can be randomly generated and need not be updated. All the hidden node parameters are independent of the target functions or the training datasets. Benefitting from the tuning-free framework, ELM not only learns up to thousand times faster than conventional gradient descent methods for SLFNs and the support vector machine (SVM) but also preserves a reasonable generalization performance. For most applications, it has been shown that the learning phase of ELM is almost real-time processing in an ordinary PC. Therefore, ELM shows superiority over conventional gradient based methods and SVM on high dimensional applications and large data processing. This is especially important since nowadays data are explosive with the rapid development of the Internet, computer, and electronic equipment.

In the light of these considerations, this special issue was launched. We received papers from worldwide researchers in interdisciplinary research fields and high quality peer reviewed papers are selected to include in this special issue.
An overview paper which summarizes the recent developments on ELM and its applications in high dimensional and large data processing presented by the Guest Editors J. Cao and Z. Lin is also published in the special issue. These papers present the recent developments in ELM algorithms applications in high dimensional and large data. The accepted papers can be broadly categorized into six groups: image and video processing, target tracking and predictions, control and estimation application, ELM algorithms, web and document applications, and other applications. In the following, we give a brief description of the papers in each of the six groups.

In image and video processing, W. Xiao and Y. Lu developed a highly efficient approach for human activity recognition based on ELM and using only one triaxial accelerometer. S. Ding et al. combined multilayer ELM (MLELM) and ELM with kernel (KELM) to put forward deep extreme learning machine (DELM) and apply it to EEG classification. T. Xiao et al. proposed a novel automatic anomaly detection approach with ELM-based visual attribute and Spatiotemporal Pyramid (STP). Y. Jiang et al. developed a new boosting ELM named MAELM, which applies the Multiclass AdaBoost in ELM ensemble to directly solve 
multiclass classification problem. Z. Chang et al. utilized the three-dimensional motion history image and the ELM to realize the recognition of body movement trajectory robustly, efficiently, and accurately.

In target tracking and predictions, X. Jiang et al. proposed fusion location framework with particle filter using Wi-Fi signals and motion sensors. The ELM regression algorithm is used to predict position based on motion sensors, and Wi-Fi fingerprint location is used to solve the error accumulation of motion sensors. J. Cao et al. adopted the ELM-based predictors for the prediction of porosity and permeability in heterogeneous sandstone reservoir of Permian formation in Yanqi survey. P. Zhou et al. developed a data-driven dynamic modeling method for online prediction of silicon content using improved ELM with the help of principle component analysis (PCA). W. Mao et al. proposed an online sequential ELM with the new weighted strategy for nonstationary time series prediction. This strategy ranks the samples' importance by means of the Leave-One-Out (LOO) error of each new added sample and then assigns various weights. Y. Zhao et al. investigated an active service quality prediction method based on ELM by concise feature extraction. An efficient prefix tree based mining algorithm together with some effective pruning rules is developed to mine the EC rule.

In control and estimation applications, D. Xiao and J. Wang developed a mean value staged kernel ELM combining with the partial least square method (KELM-PLS) to optimize the piercing efficiency and energy consumption prediction model. Y. Mao et al. proposed an online sequential ELM dynamic recursive partial least square approach (OS-ELMDRPLS) to establish a prediction model for the final temperature of a tube billet. W. Chu et al. applied the original ELM to estimate train dwell time in urban rail traffic. P. K. Wong et al. employed the kernel ELM algorithm as a model predictive controller to build a backup air-ratio model. D. Li et al. introduced a new quasilinear model based ELM (QLELM) algorithm for internal model control with nonlinearity. Y. Zhang et al. proposed a novel intelligent control design based on ELM to assist the control of a quadrotor helicopter.

In developments of ELM algorithm, P. Zhang and Z. Yang developed a new hybrid machine learning method called robust AdaBoost.RT based ensemble ELM (RAEELM) for real-world regression problems. C. Zhu et al. proposed a distance based multiple kernel ELM (DBMKELM), which provides a two-stage multiple kernel learning approach with high efficiency. B. Jia et al. presented the twodimensional ELM (2DELM) by dealing with matrix data directly. Unlike original ELM that handles vectors, 2DELM takes the matrices as input features without vectorization. Q. Leng et al. proposed a simple and efficient one-class classifier based on ELM to tackle the slow learning speed in autoencoder neural network. Y. Qu and A. Deng implemented the evolutionary local coupled ELM (ELC-ELM) to further refine the weight searching space of LC-ELM. Y. Liu et al. developed a novel particle swarm optimization based selective ensemble (PSOSEN) of online sequential ELM.

In web and document applications, H. Liu and Y. Li applied a new feature to analyze user behavior and information dissemination and used ELM to predict behaviors of users. Y. Zhao et al. proposed a keyword search measure on probabilistic extensible markup language (XML) data based on ELM. B. Wang et al. constructed feature space to describe features of every two programs with possible plagiarism relationship by employing ELM. X. Bi et al. presented a solution to the distributed learning over massive XML documents. The new method provides distributed conversion of XML documents into the representation model in parallel based on MapReduce. A distributed learning component based on ELM is then used for mining tasks of classification or clustering.

In other applications with ELM, H. Gao et al. investigated two sample-based ELM algorithms in handling the problem of missing data in regressions and classifications. L. Ding et al. developed an efficient ELM-based two stages query processing optimization model for big data. S. Zhang et al. utilized the modified ELM approach to develop a soft-sensing method for online detection of the alumina concentration. X. Ding and M. Lei combined the constrained-optimization based ELM with the rough set theory for military data classification and labeling.

\section{Acknowledgments}

As the Guest Editors of the special issue, we would like to express our gratitude to all the authors who have contributed their work to our special issue. We also would like to express our sincere appreciation to the reviewers for their valuable and insightful comments on the submitted manuscripts to our special issue.

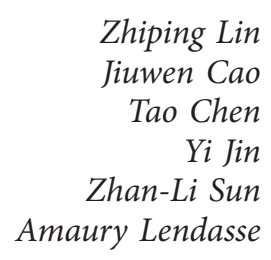

Zhiping Lin Jiuwen Cao Chen Yi Jin Amaury Lendasse 


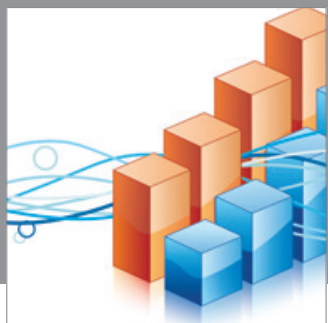

Advances in

Operations Research

mansans

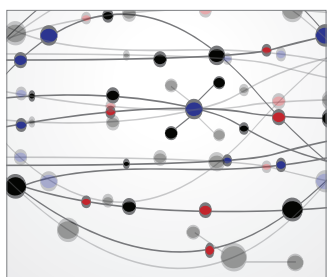

The Scientific World Journal
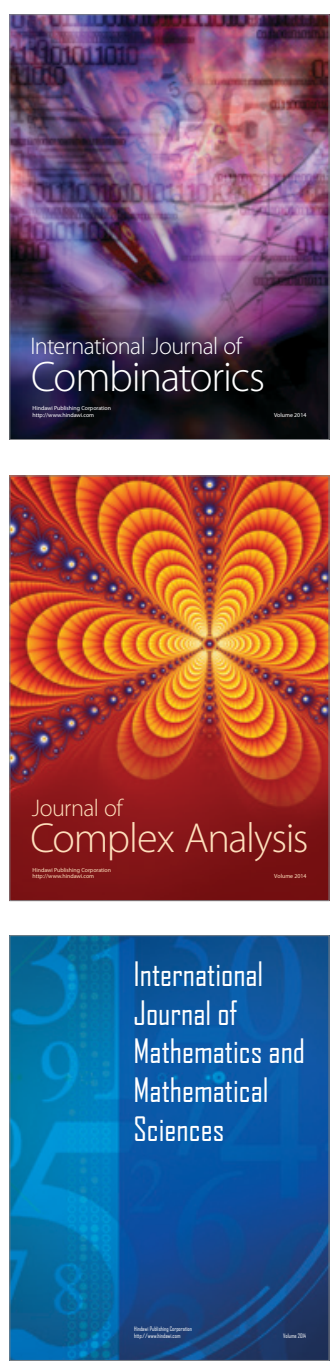
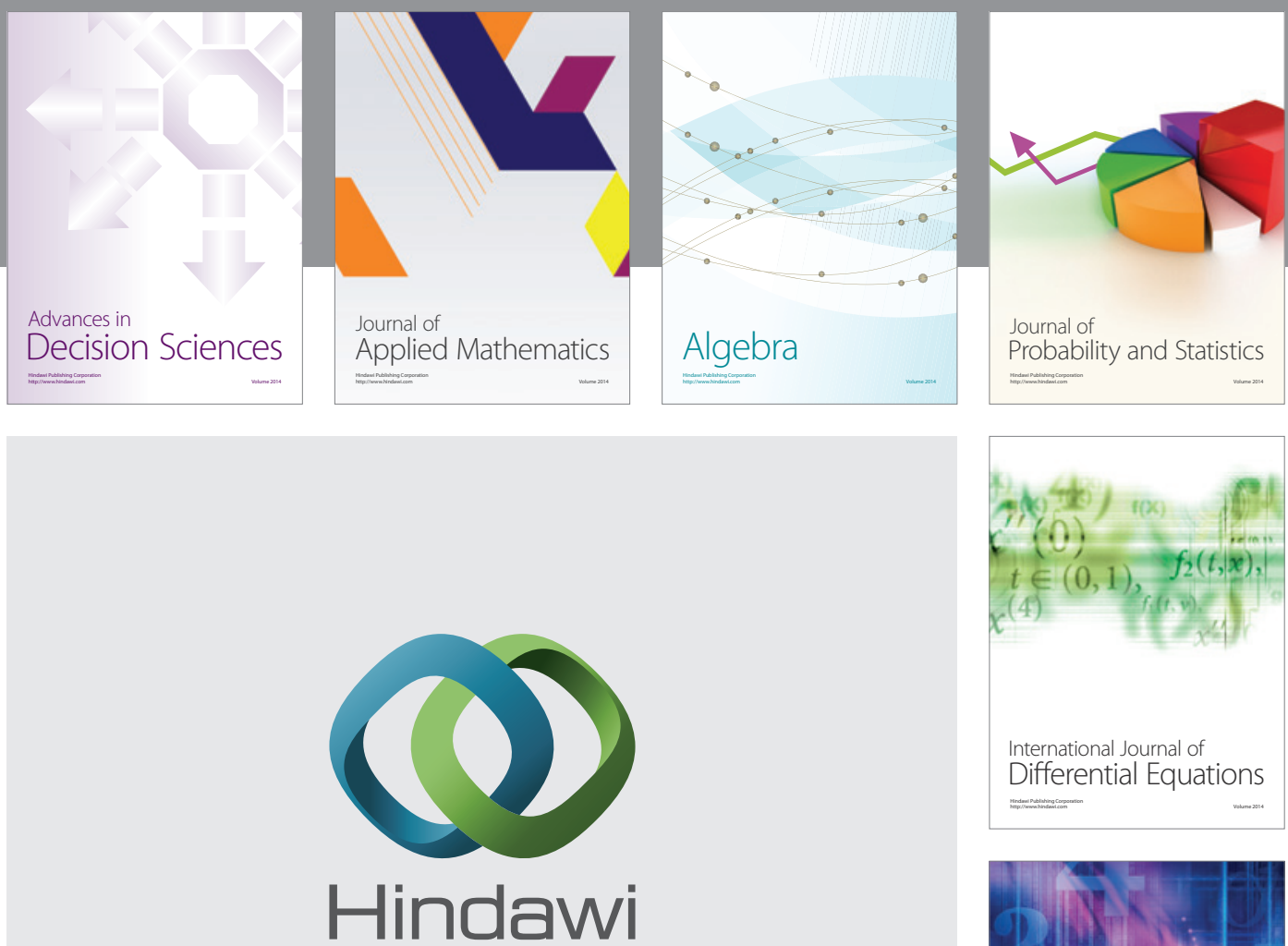

Submit your manuscripts at http://www.hindawi.com
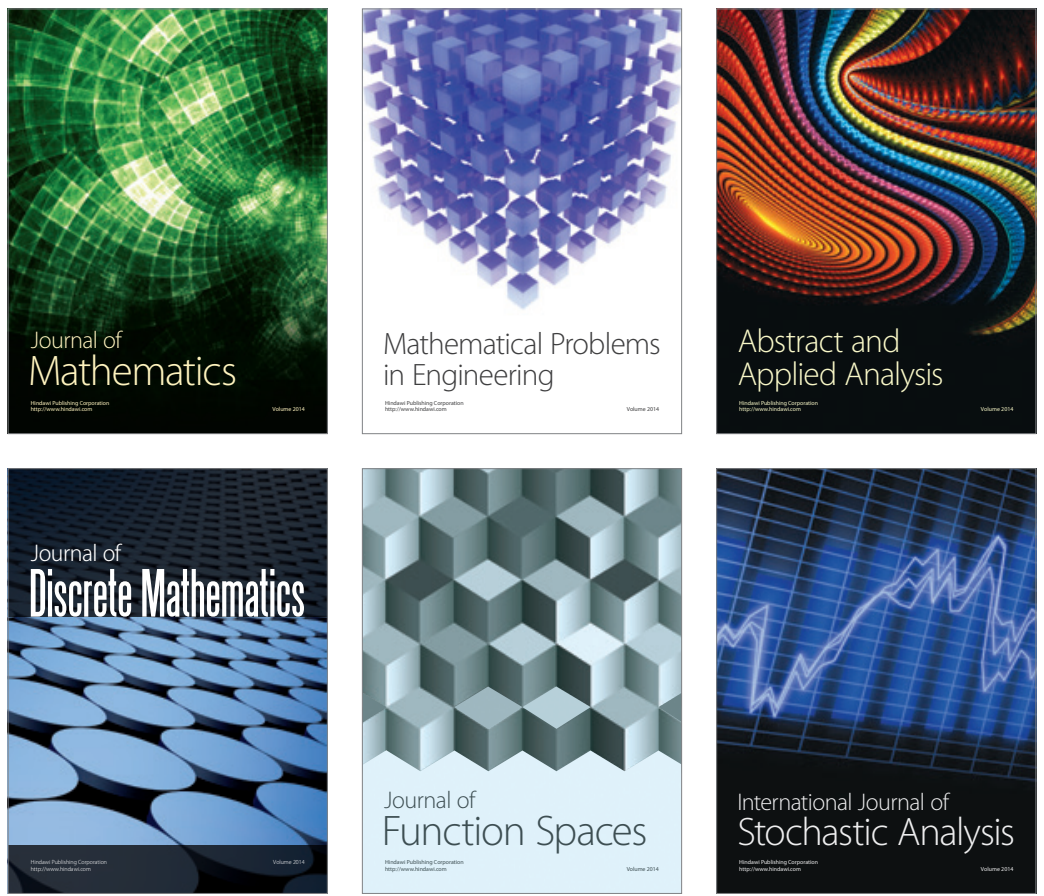

Journal of

Function Spaces

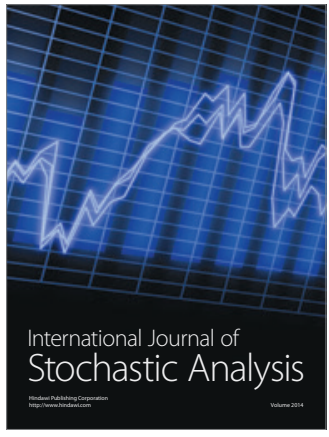

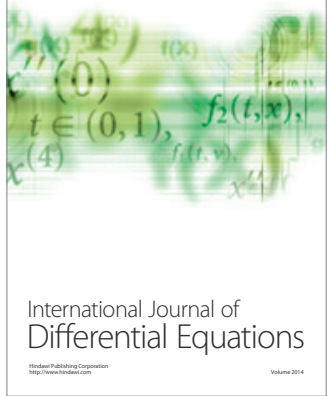
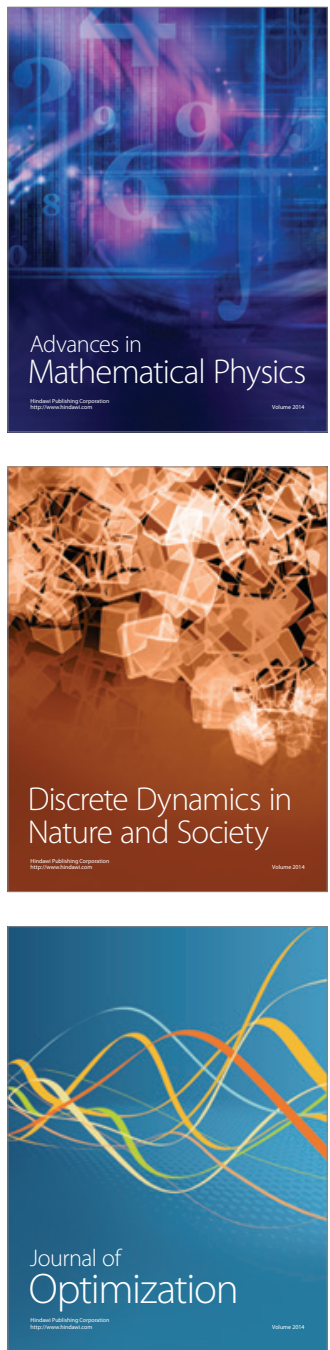